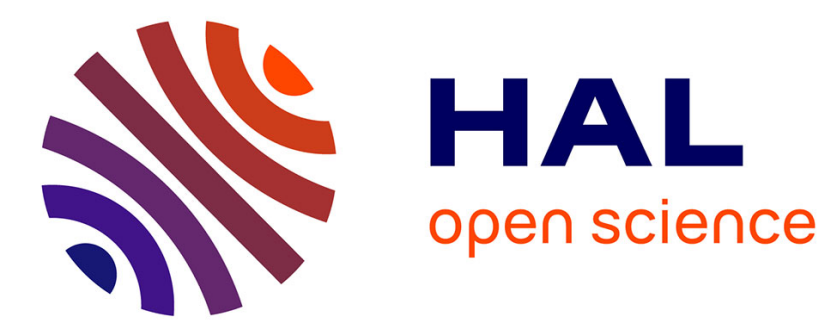

\title{
Interreflections in Computer Vision: A Survey and an Introduction to Spectral Infinite-Bounce Model
}

\author{
Rada Deeb, Damien Muselet, Mathieu Hébert, Alain Trémeau
}

\section{To cite this version:}

Rada Deeb, Damien Muselet, Mathieu Hébert, Alain Trémeau. Interreflections in Computer Vision: A Survey and an Introduction to Spectral Infinite-Bounce Model. Journal of Mathematical Imaging and Vision, 2018. hal-01900392

\section{HAL Id: hal-01900392 \\ https://hal.science/hal-01900392}

Submitted on 22 Oct 2018

HAL is a multi-disciplinary open access archive for the deposit and dissemination of scientific research documents, whether they are published or not. The documents may come from teaching and research institutions in France or abroad, or from public or private research centers.
L'archive ouverte pluridisciplinaire HAL, est destinée au dépôt et à la diffusion de documents scientifiques de niveau recherche, publiés ou non, émanant des établissements d'enseignement et de recherche français ou étrangers, des laboratoires publics ou privés. 


\title{
Interreflections in Computer Vision
}

\section{A Survey \& An Introduction To Spectral Infinite-Bounce Model}

\author{
Rada Deeb • Damien Muselet • Mathieu Hebert • Alain Tremeau
}

\begin{abstract}
Interreflections are observed on concave objects or when multiple objects are located closely. In a vision system, interreflections can largely affect color values captured by the camera. Due to this fact, modeling interreflections is important for many vision applications. In this paper, we consider the problem of treating and modeling interreflections in the domain of computer vision. First, a survey of existing approaches in the state of the art is given. These approaches are detailed, discussed and compared. Most of the state of the art models take into consideration only two bounces of light between surface elements. We, afterwards, introduce a new interreflection model based on radiometric definitions. This model is the first one that takes into consideration an infinite number of light bounces between surface elements while providing image RGB values as a result. The accuracy of our model is studied by comparing it to real camera outputs. Thanks to our new model, the importance of using infinite bounces of light while studying interreflection, instead of only two bounces is demonstrated.
\end{abstract}

Keywords Interreflection - Mutual illumination . Inverse problem · Computer vision

\section{R. Deeb}

Université de Lyon, CNRS, Institut d'Optique Graduate School, Laboratoire Hubert Curien UMR 5516, F-42023, Saint-Etienne, France

E-mail: rada.deeb@univ-st-etienne.fr

D.Muselet · M.Hebert · A.Tremeau

Université de Lyon, CNRS, Institut d'Optique Graduate School, Laboratoire Hubert Curien UMR 5516, F-42023, Saint-Etienne, France

\section{Introduction}

The scientific study of color has a long history: many theories, recommendations and tools have been proposed for its assessment, measurement, simulation or reproduction, with permanent improvement attempts. Most of the time, the question of color is addressed independently of the object's surrounding, except in image synthesis where the whole scene is taken into account in the representation of the object, adding more and more realistic details, especially the specular reflection of the scene on the object when it has a glossy surface. Similar reflection phenomena also occur on mate, diffusing surfaces, even though it is generally less striking visually, and therefore often ignored.

When a scene containing concave surfaces is illuminated, light strikes successively different surface elements before reaching the sensor. This is what we call interreflection (or mutual illumination). Appearance of objects in a scene can be considerably altered by interreflection as it affects the perceived color of their surface elements. However, most of the existing vision systems neglect the effect of interreflections, leading often to erroneous results. Physically speaking, photons received from the light source by a surface element can bounce towards a second surface element, then a third, and so on, until they reach the detector. The power of the rays is reduced after each bounce, and their spectral distributions are modified according to the spectral bidirectional reflectance distribution function of the successive surface elements they hit.

In computer graphics, this process has been simulated iteratively by using different approaches in order to calculate the color values of each surface element of the scene to be displayed on a screen [1-3]. On the other hand, in computer vision, researchers are inter- 
ested in the challenging inverse problem: how to find illuminant spectral power distribution (SPD), surface spectral reflectance, or both from the pixels values in an image. Moreover, many vision applications need to retrieve color constancy of scene's objects in order to give satisfactory results, in this case removing the effect of interreflection is necessary.

In the end of 1980s and the early 1990s, many studies on interreflection for the sake of different computer vision applications were published in the literature [410]. These studies were generally focused on the understanding of this phenomenon and on finding models that suit it. The main applications for this research were in shape from shading $[4,6,10]$ and color constancy domains [7-9]. Nevertheless, the research in this area has become less popular recently, focusing mainly on the adaptation of early proposed models for different types of applications and needs [11-13]. This seems to follow a general trend in computer vision, as recent research in this area is focusing more on pattern recognition approaches and less on physics-based approaches.

This paper consists of two major parts, the first is a detailed survey of the main existing approaches which aim to model interreflections in computer vision applications. The main existing approaches are compared based on different criteria such as the number of bounces, the number of color channels or wavebands, and the required assumptions. The second part is a new spectral infinite-bounce model of interreflection for Lambertian surfaces. This model is more general than the ones proposed earlier in the literature. It links RGB values in the image to a physical spectral model of interreflection taking into consideration an infinite number of light bounces. This can be particularly helpful for the research process in the domain of computer vision. We aim to give a fresh restart to interreflection modeling in computer vision based on a physical study of this phenomenon. We believe that understanding image values from an optical point of view can be very helpful, and can be used side by side with pattern recognition approaches.

The paper starts with a presentation of the main used terms and notations and ends with an evaluation of the proposed model and a discussion of its advantages and limitations.

\section{Definitions and Notations}

Having a point $P_{i}$ of the scene $\mathcal{S}$, the surface of the infinitesimal facet represented by this point is denoted as $d P_{i}$.

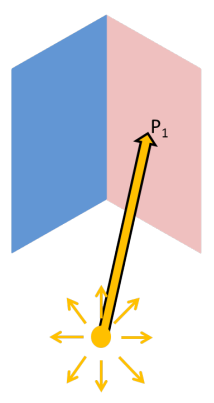

(a)

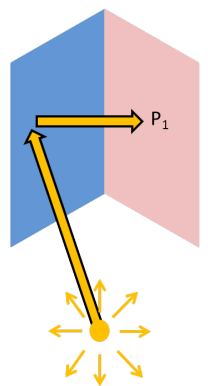

(b)

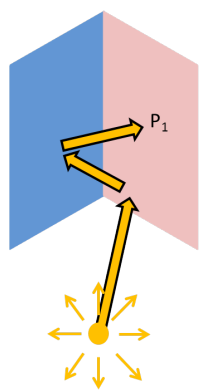

(c)
Fig. 1: Decomposition of the irradiance received by $P_{1}$ : (a) Direct light, (b) first bounce of interreflection corresponding to what we call a two-bounce model, (c) second bounce of interreflection. For the sake of clarity, the sums over all the pixels (multiple arrows) are not illustrated here.

In radiometry, the radiance, $L$, is the density of radiant power, $F$, per unit of geometrical extent, $G$ [14]:

$L=d^{2} F / d^{2} G$,

where $d^{2} G$ is the elemental geometrical extent ${ }^{1}$ between two elemental areas $d P_{i}$ and $d P_{j}$, around two points (distant enough from each other) $P_{i}$ and $P_{j}$, respectively, and is defined as in [15]:

$d^{2} G=\frac{d P_{i} \cos \theta_{i} d P_{j} \cos \theta_{j}}{\Delta^{2}}$,

where $\Delta$ is the euclidean distance between the centroids of the two surfaces, $\theta_{i}$ and $\theta_{j}$ are the angles between $P_{i} P_{j}$ and the normals of the facets represented by $P_{i}$ and $P_{j}$ respectively (Figure 2 ).

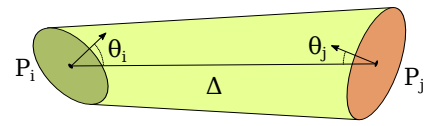

Fig. 2: Geometrical extent between two elemental areas.

The irradiance, $E$, is the density of radiant power per unit area, $A$ :

$E=d F / d A$.

Based on these definitions, the irradiance can be written in terms of radiance as follows:

$d E=L d G / d A$.

The irradiance received at a point $P_{i}$ is denoted as $E\left(P_{i}\right)$, and the radiance from $P_{i}$ to a second point $P_{j}$ is

1 Also called étendue 
denoted as $L\left(P_{i}, P_{j}\right)$. For lambertian surfaces, the radiance is independent of the propagation direction, then the radiance of $P_{i}$ is denoted as $L\left(P_{i}\right)$, and, according to Lambert's law, it can be defined as:

$L\left(P_{i}\right)=E\left(P_{i}\right) r_{i} / \pi$,

where $r_{i}$ is the reflectance of the point $P_{i}$.

Along this paper, direct irradiance received from the light source, and radiance after the first bounce of direct light are denoted as $E_{0}$ and $L_{1}$ respectively. The notations $\mathbf{L}$ and $\mathbf{E}$ refer to, respectively, the vectors of radiance and irradiance on various surface elements: $\mathbf{L}=$ $\left[\begin{array}{lll}L\left(P_{1}\right) & L\left(P_{2}\right) & \ldots\end{array}\right]^{T}$ and $\mathbf{E}=\left[\begin{array}{lll}E\left(P_{1}\right) & E\left(P_{2}\right) & \ldots\end{array}\right]^{T}$.

Actually, the terms $L\left(P_{i}\right), E\left(P_{i}\right)$ and $r_{i}$ are all wavelength dependent, but when used in this paper they are taken for only a single wavelength. However, when talking about spectral functions, the terms $R(\lambda), L(\lambda)$ and $E(\lambda)$ are used for spectral reflectance, spectral radiance, and spectral irradiance respectively.

By defining the response function, $C_{k}$ for each camera sensor $k$, the $k$-th sensor response, when observing a lambertian surface of spectral reflectance $R(\lambda)$ and lighted by an illuminant $E(\lambda)$, can be written as:

$\rho^{k}=\frac{1}{\pi} \int_{\lambda} E(\lambda) R(\lambda) C^{k}(\lambda) d \lambda$

\section{State of the Art}

Many methods have been proposed in the literature in order to model interreflections in computer vision applications. Some approaches focus on removing the effect of interreflections $[6,10-13,16]$, whereas others use it as extra information that may help in solving other problems such as light SPD estimation or surface spectral reflectance estimation [9]. Moreover, the proposed systems differ in many ways regarding the number of color channels or wavelengths, the number of light bounces they handle, and the number and the type of assumptions they pose:

Number of wavelengths or color channels. While some methods take into consideration only a single wavelength either by using a narrow-band light or by handling monochromatic surfaces $[6,11,12]$, others propose a more general approach by working in RGB color space $[10,16]$. Moreover, there exist some methods that handle the set of visible wavelengths leading to a spectral approach [9].
Number of bounces. There exist two-bounce approaches claiming that taking into consideration only one bounce of interreflection is enough in most cases $[9,10,12]$. However, some of these methods propose an extension to handle more light bounces [12]. Other methods treat directly an infinite number of bounces $[6,11,16]$.

Assumptions. Handling interreflections is often an issue. Thus, assumptions or preprocessing can be required to make this task treatable. Some assumptions are upon the spectral reflectances of the surfaces, for example the use of uniformly colored surfaces $[9,10,12,17]$. Others are light-related regarding the number of light sources, and their spectral power distributions $[9,12,16]$. Moreover, some approaches depend on other methods such as the dependence on a shape from shading method $[6,16]$. Others need some preprocessing steps such as light field calculation [11] or some calibration steps to estimate parameters needed for the system [12].

In the following sections, we give a detailed presentation of the main approaches reported above. We organized them according to the used methodology to treat interreflection. A comparative table can be found in Table 1.

\subsection{Spectral Analysis of Interreflections for Color Constancy}

Funt, Drew and Ho [7-9] proposed to use interreflections as additional information to find illumination spectral power distribution and surface spectral reflectance from RGB data. Considering that some parts of the surface are not affected by interreflections, they combine equations with and without interreflections to solve them for the unknown parameters. Their work is based on modeling the spectral reflectance and the spectral power distribution of lights by finite dimensional linear models using a set of basis functions. Then, they solve a set of equations with equal number of unknown parameters. They demonstrate that it is possible to use three basis functions for surfaces and illumination with no need for more than three sensor classes.

Considering diffuse irradiance $E(\lambda)$, and two lambertian surfaces whose spectral reflectances are $R^{(1)}(\lambda)$ and $R^{(2)}(\lambda)$ respectively, radiances, $L^{(1)}(\lambda)$ and $L^{(2)}(\lambda)$, reflected from surface 1 and 2 , without taking into consideration interreflections, can be written as:

$$
\begin{aligned}
L^{(1)}(\lambda) & =\frac{1}{\pi} E(\lambda) R^{(1)}(\lambda), \\
L^{(2)}(\lambda) & =\frac{1}{\pi} E(\lambda) R^{(2)}(\lambda) .
\end{aligned}
$$


Table 1: A comparison table of state of the art approaches

\begin{tabular}{|c|c|c|c|c|c|c|}
\hline Approach & $\begin{array}{l}\text { Spectral/No. } \\
\text { color channels }\end{array}$ & $\begin{array}{l}\text { No. } \\
\text { Bounces }\end{array}$ & No. illuminants & $\begin{array}{l}\text { No. spectral } \\
\text { reflectances }\end{array}$ & $\begin{array}{l}\text { Known } \\
\text { Geometry }\end{array}$ & Dependency on \\
\hline Nayar et al.. [6] & 1 & $\infty$ & 1 & Multi & No & Shape from shading \\
\hline Funt et al. [9] & Spectral 2 & 2 & 1 & 2 & No & - \\
\hline Nayar and Gong [16] & 3 & $\infty$ & 1 & Multi & No & Shape from Shading \\
\hline Funt and Drew [10] & 3 & 2 & 1 & 2 & No & - \\
\hline Seitz et al. [11] & 1 & $\infty$ & 1 & Multi & No & ISF matrix \\
\hline Liao et al. [12] & 1 & $2+$ & $2+$ & 1 & No & 2 Calibration steps \\
\hline Our model & Spectral & $\infty$ & 1 & Multi & Yes & - \\
\hline
\end{tabular}

By modeling the illumination using $u$ basis functions denoted as $E_{i}$, the spectral power distribution can be written as:

$E(\lambda)=\sum_{i=1}^{u} \epsilon_{i} E_{i}(\lambda)$

Similarly, the two surfaces' spectral reflectances are modeled with $v$ basis functions denoted as $R_{j}$ :

$R^{(1)}(\lambda)=\sum_{j=1}^{v} \sigma_{j}^{(1)} R_{j}(\lambda)$,
$R^{(2)}(\lambda)=\sum_{j=1}^{v} \sigma_{j}^{(2)} R_{j}(\lambda)$.

Using these equations, we can rewrite (7) as:

$L^{(1)}(\lambda)=\frac{1}{\pi} \sum_{i=1}^{u} \sum_{j=1}^{v} \epsilon_{i} \sigma_{j}^{(1)} E_{i}(\lambda) R_{j}(\lambda)$,

$L^{(2)}(\lambda)=\frac{1}{\pi} \sum_{i=1}^{u} \sum_{j=1}^{v} \epsilon_{i} \sigma_{j}^{(2)} E_{i}(\lambda) R_{j}(\lambda)$.

Notice that, in these equations, the unknown parameters are $\epsilon_{i}, \sigma_{j}^{(1)}$ and $\sigma_{j}^{(2)}$, so we have $2 v+u$ unknown parameters.

In order to have more equations, the authors propose to take into consideration regions affected by interreflections. In computer graphics, diffuse interreflections are modeled using configuration factors which give the fraction of light from a surface that reaches the other surfaces. Let us consider $\alpha_{12}$ the fraction of light signal reflected from a surface 1 to a surface 2 , and $\alpha_{21}$ the fraction of light signal reflected from surface 2 to surface 1 . Let $L_{m}^{j}$ be radiance reflected in every direction by one of the two surfaces $(j=1$ or 2$)$ in the mutual reflection area, thus it can be written as:
$L_{m}^{(1)}(\lambda)=L^{(1)}(\lambda)+\alpha_{21} L_{m}^{(2)}(\lambda) R^{(1)}(\lambda)$,

$L_{m}^{(2)}(\lambda)=L^{(2)}(\lambda)+\alpha_{12} L_{m}^{(1)}(\lambda) R^{(2)}(\lambda)$.

By developing these equations, we obtain:

$L_{m}^{(1)}(\lambda)=\frac{L^{(1)}(\lambda)+\alpha_{21} L^{(2)}(\lambda) R^{(1)}(\lambda)}{1-\alpha_{12} \alpha_{21} R^{(1)}(\lambda) R^{(2)}(\lambda)}$,

$L_{m}^{(2)}(\lambda)=\frac{L^{(2)}(\lambda)+\alpha_{12} L^{(1)}(\lambda) R^{(2)}(\lambda)}{1-\alpha_{12} \alpha_{21} R^{(1)}(\lambda) R^{(2)}(\lambda)}$.

In order to make these equations easier to solve numerically, the authors assume that the dominator is equal to one. They suggest that this can be done without significant loss in accuracy in most cases. That's because both $\alpha$ and $R(\lambda)$ are inferior to one. Thus, the equations can be rewritten as:

$L_{m}^{(1)}(\lambda)=L^{(1)}(\lambda)+\alpha_{21} L^{(2)}(\lambda) R^{(1)}(\lambda)$,

$L_{m}^{(2)}(\lambda)=L^{(2)}(\lambda)+\alpha_{12} L^{(1)}(\lambda) R^{(2)}(\lambda)$.

However, for highly reflective materials (high values of $R(\lambda)$ ), and for geometric configurations with high values of $\alpha$, this approximative model is far from being accurate.

Moreover, with this latter assumption, only one bounce of interreflection is considered, further bounces being ignored; therefore, the previous equations represent a two-bounce model.

From the previous equations, knowing that:

$L^{(1)}(\lambda) R^{(2)}(\lambda)=L^{(2)}(\lambda) R^{(1)}(\lambda)$,

the ratio between $\alpha_{12}$ and $\alpha_{21}$ can be written as:

$\frac{\alpha_{21}}{\alpha_{12}}=\frac{L_{m}^{(1)}(\lambda)-L^{(1)}(\lambda)}{L_{m}^{(2)}(\lambda)-L^{(2)}(\lambda)}$.

2 Each spectral function is modeled with three basis functions. 
This ratio can be known as all the radiances are considered to be measurable, thus, only one unknown parameter is added with two additional equations (13).

In order to solve the set of equations (10) and (13), let us first consider the sensor sensitivity function $C_{k}(\lambda)$ for $k=1 \ldots p$ where $p$ is the number of sensors. Then, by integrating according to the wavelength over the visible spectrum of light, we can define the following precalculated tensor:

$g_{i j k}=\frac{1}{\pi} \int_{\lambda} E_{i}(\lambda) R_{j}(\lambda) C_{k}(\lambda) d \lambda$.

By rewriting equations (10) and (13) in terms of camera response, we can write the $k$-th sensor response, $\rho_{k}$, as follows:

$\rho_{k}^{(1)}=\sum_{i=1}^{u} \sum_{j=1}^{v} \epsilon_{i} \sigma_{j}^{(1)} g_{i j k}$,

$\rho_{k}^{(2)}=\sum_{i=1}^{u} \sum_{j=1}^{v} \epsilon_{i} \sigma_{j}^{(2)} g_{i j k}$,

for the response of the camera to the radiance reflected after one light bounce on surfaces 1 and 2, and:

$\rho_{k}^{m(2)}=\rho_{k}^{(2)}+\alpha_{12} \sum_{i=1}^{u} \sum_{j=1}^{v} \sum_{j^{\prime}=1}^{v} \epsilon_{i} \sigma_{j}^{(1)} \sigma_{j^{\prime}}^{(2)} h_{i j j^{\prime} k}$,

for the camera response in the mutual reflection area taking into consideration only one bounce of interreflection, where:

$h_{i j j^{\prime} k}=\frac{1}{\pi} \int_{\lambda} E_{i}(\lambda) R_{j}(\lambda) R_{j^{\prime}}(\lambda) C_{k}(\lambda) d \lambda$.

Now, the set of equations (17), (18) and (19) for the unknown parameters: $\epsilon_{i}, \sigma_{j}^{(1)}, \sigma_{j}^{(2)}$ and $\alpha$ needs to be solved. Representing light spectral power distribution and spectral reflectances using three basis functions for each, will lead to 10 unknowns. However, having three sensors, and three equations, we have only 9 equations. Then, in order to be able to solve this problem and use only three sensors, the authors impose that $\epsilon_{1}=1$, thus neglecting the brightness of the surfaces.
As we have seen above, the idea behind this work is to use interreflections in order to obtain the spectral power distribution of illuminant and the spectral reflectance of surfaces. This is possible by supposing that there is at least one point on each surface without the effect of interreflections. In addition, the authors studied the convergence and uniqueness of their solution, and they provided a discussion on how the parameter $\alpha$ varies with the angle between the two surfaces and the relative position.

This approach gives an approximation of the spectral reflectance of surfaces and the spectral power distribution of light based only on one RGB image. Moreover, no calibration or pre-processing is needed. However, only ambient light is considered (isotropic diffuse illumination). This assumption is made in order to consider the irradiance at each point of both surfaces to be the same. In addition, the method considers only two convex lambertian surfaces with uniform spectral reflectances. The existence of at least one point on each surface with no mutual illumination is needed. The authors also suppose that taking into consideration only two light bounces is sufficient, which is not the case for all scenes.

Later, this work has been extended to a variational approach in order to relax some constraints, especially those related to diffuse illuminant and to the number of surfaces. However, the complexity of the algorithm has considerably increased and the model still handles only one bounce of interreflection [18].

\subsection{Color space analysis of interreflections}

In a more recent work [10], Funt and Drew focused their attention on an analysis in RGB color space to separate the color with no interreflection from the color after one bounce of interreflection. The configuration is similar to the previous work: two homogeneous color flat surfaces. The light is assumed to be constant regarding its spectral power distribution but its irradiance can vary with position.

The main idea behind this work is that a plane in RGB space can be formed from two colors: the color after one bounce of light, i.e. without taking into consideration interreflection, called one-bounce color, and the color after two bounces of light, called two-bounce color. Then, the intersection between the two planes corresponding to the two surfaces gives the two-bounce RGB color.

In a two-bounce model, the radiance received by an area on the first surface corresponding to a pixel $x$ in the image can be written as: 


$$
\begin{aligned}
& L^{(1)}(x, \lambda) \simeq \alpha^{(1)}(x) E(\lambda) R^{(1)}(\lambda) \\
& \quad+\beta^{(12)}(x) E(\lambda) R^{(1)}(\lambda) R^{(2)}(\lambda),
\end{aligned}
$$

where $\alpha^{(1)}(x)$ represents the variation of irradiance at point $x$, and $\beta^{(12)}(x)$ represents the relative contribution of interreflection to the radiance.

A similar equation can be written for the second surface.

By multiplying equation (21) with sensor spectral response and then by integrating over the visible spectrum, the previous equation can be written in terms of camera response as:

$\rho_{k}^{(1)}(x) \simeq \alpha_{1}^{(1)}(x) \rho_{k, 1}^{(1)}(x)+\beta_{1}^{(12)}(x) \rho_{k, 2}(x)$,

$\rho_{k}^{(2)}(x) \simeq \alpha_{1}^{(2)}(x) \rho_{k, 1}^{(2)}(x)+\beta_{1}^{(12)}(x) \rho_{k, 2}(x)$,

where $\rho_{k, 1}$ represents response of the sensor $k$ after one bounce of light, and can be written as:

$\rho_{k, 1}^{(1)}(x)=\frac{1}{\pi} \int_{\lambda} E(\lambda) R^{(1)}(\lambda) C_{k}(\lambda) d \lambda$,

$\rho_{k, 1}^{(2)}(x)=\frac{1}{\pi} \int_{\lambda} E(\lambda) R^{(2)}(\lambda) C_{k}(\lambda) d \lambda$,

and $\rho_{k, 2}$ represents the sensor $k$ response for the color issued from taking into consideration two bounces of light, and can be written as:

$\rho_{k, 2}(x)=\frac{1}{\pi} \int_{\lambda} E(\lambda) R^{(1)}(\lambda) R^{(2)}(\lambda) C_{k}(\lambda) d \lambda$.

From equation (22), we can see that all image values corresponding to one surface can be written as a linear combination of one-bounce and two-bounce colors, thus they lie in a plane defined by these two colors. The plane of each surface is found by singular value decomposition of all color values of this surface. The two vectors defining the plane are the first two eigenvectors.

The two-bounce color corresponds to the intersection between the two planes (corresponding to first and second surfaces). This intersection can be found by algebra. However, this method fails to find precisely the one-bounce color except under an assumption that there exists at least one point on each surface that is not affected by interreflections.

Given that one can obtain the one-bounce and twobounce colors, the original image can be easily decomposed into two components corresponding to these colors.

This approach was tested on both synthetic and real images. The authors studied some special cases where the two colors do not differ enough leading to confound the two planes into one. However, they suggested that SVD can detect this kind of cases, where no separation of these two colors is possible.

This method separates one-bounce and two-bounce colors based on RGB values taken from one image. It is fast and needs no calibration or preprocessing. However, only one bounce of interreflection is taken into consideration, and each surface needs to have a uniform spectral reflectance. Spectral power distribution of lighting needs to be uniform in all directions. Moreover, this approach failed to suggest an upper bound to the one-bounce color, so it assumes the existence of at least one point on each surface that is not affected by interreflections. A real world scene satisfying this assumption is rather hard to meet.

\subsection{Radiosity-Based Approaches}

None of the previously presented approaches tried to provide a model for interreflections. However, the majority of the existing approaches in the state of the art are based on a physical definition of interreflections, presented in this section, providing a rigorous model to handle interreflections in computer vision applications.

\subsubsection{Continuous model}

Inspired by radiosity equation in computer graphics [1], Koenderink and Van Doorn [19] introduced an interreflection equation for Lambertian surfaces based on a definition of a geometrical kernel. Different approaches in the literature, especially in shape from shading research field, are based on this equation. Based on the works by Koenderink and Van Doorn [19], and by Forsyth and Zisserman $[4,5,20]$ and Nayar et al. [6], the interreflection equation can be written, using the notations and definitions introduced in Section 2, as:

$L\left(P_{i}\right)=L_{1}\left(P_{i}\right)+\frac{r_{i}}{\pi} \int_{P_{j} \in \mathcal{S}} K\left(P_{i}, P_{j}\right) L\left(P_{j}\right) d P_{j}$,

where $\mathcal{S}$ is the area containing all surface elements of the scene, and $K\left(P_{i}, P_{j}\right)$ is the geometrical kernel (or interreflection kernel), driven from the definition of the geometrical extent subtended by any pair of points $P_{i}$ and $P_{j}$ in the scene, defined as:

$K\left(P_{i}, P_{j}\right)=\frac{\left(\overrightarrow{N_{i}} \cdot \overrightarrow{P_{i} P_{j}}\right)\left(\vec{N}_{j} \cdot \overrightarrow{P_{j} P_{i}}\right) V\left(P_{i}, P_{j}\right)}{\Delta^{4}}$, 
where $\vec{N}_{i}$ and $\vec{N}_{j}$ are the normals of surface elements $i$ and $j$ respectively, the dot operator . is the dot product between two vectors, $\Delta$ is the euclidean distance between the two points, and $V\left(P_{i}, P_{j}\right)$ is a visibility function giving 1 when the two points can see each other and 0 otherwise, it can be defined as follows:

$V\left(P_{1}, P_{2}\right)=\frac{\overrightarrow{N_{1}} \cdot \overrightarrow{P_{1} P_{2}}+\left|\overrightarrow{N_{1}} \cdot \overrightarrow{P_{1} P_{2}}\right|}{2\left|\overrightarrow{N_{1}} \cdot \overrightarrow{P_{1} P_{2}}\right|} \cdot \frac{\overrightarrow{P_{2} P_{1}}+\left|\overrightarrow{N_{2}} \cdot \overrightarrow{P_{2} P_{1}}\right|}{2\left|\overrightarrow{N_{2}} \cdot \overrightarrow{P_{2} P_{1}}\right|}$.

Equation (26) is driven from the definition of the geometrical extent, (see equation (2)). The reason behind that $\Delta$ is raised to the power 4 instead of the power 2 , is the use of dot products instead of $\cos \theta_{i}$ and $\cos \theta_{j}$ in the numerator.

Note that this interreflection equation stands only for Lambertian surfaces as the radiance is assumed to be the same in all directions: $L\left(P_{j}, P_{i}\right)=L\left(P_{j}\right)$.

Equation (25) does not have a straightforward solution for $L\left(P_{i}\right)$. However, an analytical solution to this equation exists in case of uniformly colored surfaces [19], by modeling infinite bounces of direct radiance, using Neumann series:

$L\left(P_{i}\right)=L_{1}\left(P_{i}\right)+\sum_{b=1}^{\infty} r^{b} \int_{P_{j} \in \mathcal{S}} K_{b}\left(P_{i}, P_{j}\right) L_{1}\left(P_{j}\right) d P_{j}$,

where $b$ denotes the number of bounces,

$K_{b}\left(P_{i}, P_{j}\right)=\int_{P_{k} \in \mathcal{S}} \frac{K\left(P_{i}, P_{k}\right)}{\pi} K_{b-1}\left(P_{k}, P_{j}\right) d P_{k}$,

and,

$K_{1}=\frac{K}{\pi}$

The solution, valid only in the case of Lambertian surfaces with a uniform reflectance, can be computed iteratively.

\subsubsection{Discrete model}

A more general solution to the interreflection equation is proposed by Nayar et al. [6] by sampling the surface to $m$ facets. Both radiance and reflectance are considered to be constant over each facet. Considering the facets to be infinitesimally small, a facet $i$ is represented by a point $P_{i}$ and its area $\Delta P_{i}$. Then, equation (25) can be written as:
$L\left(P_{i}\right)=L_{1}\left(P_{i}\right)+\frac{r_{i}}{\pi} \sum_{j \neq i} L_{j} K_{i j}$,

where:

$K_{i j}=K\left(P_{i}, P_{j}\right) \Delta P_{j}$

Then, the authors introduced a matrix form for the geometrical kernel:

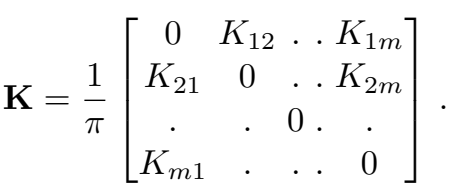

Note that this matrix is always symmetric, i.e., $K_{i j}=$ $K_{j i}$.

Moreover, as each facet has its own spectral reflectance, those for all the facets can be gathered into a diagonal matrix, denoted as $\mathbf{R}$ :

$\mathbf{R}=\left[\begin{array}{cccc}r_{1} & 0 & \ldots \ldots & 0 \\ 0 & r_{2} & \ldots . & 0 \\ . & . & . & \ldots . \\ . & . & . & \ldots \\ 0 & 0 & . . & r_{m}\end{array}\right]$

Then, equation (31) can be written as:

$\mathbf{L}=\mathbf{L}_{1}+\mathbf{R K L}$,

or:

$\mathbf{L}=(\mathbf{I}-\mathbf{R K})^{-1} \mathbf{L}_{\mathbf{1}}$,

where $\mathbf{L}$ and $\mathbf{L}_{\mathbf{1}}$ are vectors containing respectively the final radiance as perceived by the observer, and the radiance after the first bounce of direct light values on each facet.

\subsubsection{Application to shape from shading}

Nayar et al. [6] used equation (36) to iteratively correct, for gray surfaces, a pseudo shape and pre-estimated reflectance values obtained by applying a shape from shading method.

The authors' first approach handled only one wavelength. Later, they extended it to three wavebands corresponding to RGB channels [16]. They considered that the image produced by a sensor with a narrow-band filter represents reflections and interreflections of almost a monochromatic light. The shape from shading method and the recovery of actual shape using interreflections are applied separately for each color channel. 
In these approaches, both geometry and spectral reflectance are unknown and need to be estimated. Surfaces with different reflectances per position can be handled, but this method requires that the surface has no discontinuity.

\subsubsection{Extension and application to interreflection removal via light field}

In the work by Seitz et al. [11], a theoretical proof of the existence of a linear matrix transformation to obtain the first-bounce radiance from the final radiance for any scene is presented. An application of this theoretical model is also presented for lambertian surfaces for any lighting condition and without the use of a shape from shading method.

The existence of interreflection cancellation operators enables the computation of $n$-bounce images (i.e. images that would be obtained by capturing only light rays being bounced $n$ times), for each $n$, by matrix multiplication. This stands for any scene geometry and surface BRDF.

The total radiance between two points written as:

$L\left(P_{i}, P_{j}\right)=L_{1}\left(P_{i}, P_{j}\right)+L_{2}\left(P_{i}, P_{j}\right)+L_{3}\left(P_{i}, P_{j}\right)+\ldots$,

can be expressed as a light transport equation:

$$
L\left(P_{i}, P_{j}\right)=L_{1}\left(P_{i}, P_{j}\right)+\int_{k \in \mathcal{S}} A_{P_{j}}\left(P_{k}, P_{i}\right) L\left(P_{k}, P_{i}\right) .
$$

The term $A_{P_{j}}\left(P_{k}, P_{i}\right)$ is a general term: it defines the proportion of irradiance from point $P_{k}$ to $P_{i}$ that gets transported as radiance towards $P_{j}$. It is a function of the surface BRDF and of the visibility between the two points. Thus, this equation is similar to the interreflection equation (25) but it is more general as non Lambertian surfaces can be handled.

By presenting the previous equation in matrix form and developing it, we obtain:

$\mathbf{L}=(\mathbf{I}-\mathbf{A})^{-1} \mathbf{L}_{\mathbf{1}}$

Equation (36) is a special case of this equation (39). Then, for any scene, we can write:

$\mathbf{L}_{1}=\mathbf{C}_{1} \mathbf{L}$ where $\mathbf{C}_{\mathbf{1}}$ is defined as $(\mathbf{I}-\mathbf{A})$. Therefore, it is possible to remove interreflections by multiplying the radiance vector with the linear matrix $\mathbf{C}_{\mathbf{1}}$.

Moreover, the authors proved that any bounce of light can be found iteratively by treating indirect light coming from a point as if it was a direct light.

Later, the authors proposed to relate equation (39) to image intensities: for a ray $i$ with a unit radiance illuminating one point $i$ of the scene, the resulting light field $\mathbf{t}_{\mathbf{i}}$ captured by the sensor represents the full light transport in response to that ray. Vector $\mathbf{t}_{\mathbf{i}}$ is called Impulse Scatter Function (ISF). For a surface represented by $m$ points, and by concentrating the $m$ corresponding rays, we can define a matrix $\mathbf{T}$ as $\left[\begin{array}{llll}\mathbf{t}_{\mathbf{1}} & \mathbf{t}_{\mathbf{2}} & \ldots & \mathbf{t}_{\mathbf{m}}\end{array}\right]$.

The authors considered the light to be linear, then the cancellation operator can be written as:

$\mathrm{C}_{1}=\mathbf{T}_{\mathbf{1}} \mathbf{T}^{-1}$

where $\mathbf{T}_{\mathbf{1}}$ is the matrix containing Impulse Scatter Function (ISF) due to 1-bounce reflection (with no interreflections).

Note that, although $\mathbf{T}$ is measurable, $\mathbf{T}_{\mathbf{1}}$ is generally not, so this is only a theoretical proof. However, for Lambertian scenes and based on images taken at a single camera viewpoint, the cancellation operator is unique, and $\mathbf{T}_{\mathbf{1}}$ is a diagonal matrix. In addition, we can write:

$\mathbf{T}_{\mathbf{1}}[i, i]=\frac{1}{\mathbf{T}^{-1}[i, i]}$.

In the case of Lambertian surfaces, the incident and outgoing light fields are both two dimensional, ISF matrix can be captured by scanning a narrow beam of light (Laser) over the surface and capturing an image for each position of the beam.

In this work, the authors presented an important theoretical proof of the existence of a matrix operator to cancel the mutual illumination in any kind of scene. An application is provided in case of Lambertian surfaces based on light field. This approach works in any lighting condition, even challenging ones like flashlight and non uniform light sources. In addition, it is robust in case of occlusions. However, the method needs the construction of ISFs based on laser beams, which is not always easy or available for everyday applications.

\subsubsection{Application to interreflection removal without light field}

Another method to remove interreflections is proposed in [12]. The authors describe a method to separate $n$ - 
bounced light rays in order to remove the effect of interreflection. However, no light field or ISF matrix is needed. The only preconditions are: a single colored surface, and the possibility to illuminate the scene with various light sources of different SPDs.

The main idea in this approach is that, for Lambertian surfaces with uniform reflectance, whenever a light ray is bounced, its corresponding intensity in the image is attenuated by the factor $r$ corresponding to the albedo of the surface. Thus, the measured intensity value can be written as a polynomial function of $r$, and the contribution of an $n$-bounced ray can be expressed as $r^{n}$.

Based on a similar definition of the geometrical kernel as introduced before, and considering only Lambertian surfaces with a homogeneous color, the authors rewrote equation (28) as:

$L\left(P_{i}\right)=\frac{E_{0}\left(P_{i}\right)}{\pi} r+\sum_{n=1}^{\infty} r^{n} \int_{j \in \mathcal{S}} K_{n}\left(P_{i}, P_{j}\right) \frac{r E_{0}\left(P_{j}\right)}{\pi} d P_{j}$.

This equation can be seen as a polynomial function of $r$, and can be rewritten as:

$L\left(P_{i}\right)=\frac{E_{0}\left(P_{i}\right)}{\pi} r+\sum_{n=2}^{\infty}\left(\int_{j \in \mathcal{S}} K_{n-1}\left(P_{i}, P_{j}\right) \frac{E_{0}\left(P_{j}\right)}{\pi} d P_{j}\right) r^{n}$.

By defining:

$$
\begin{aligned}
C_{1}\left(P_{i}\right) & =\frac{E_{0}\left(P_{i}\right)}{\pi}, \\
C_{2}\left(P_{i}\right) & =\int_{j \in \mathcal{S}} K_{1}\left(P_{i}, P_{j}\right) \frac{E_{0}\left(P_{j}\right)}{\pi} d P_{j}, \\
\ldots & \\
C_{n}\left(P_{i}\right) & =\int_{j \in \mathcal{S}} K_{n-1}\left(P_{i}, P_{j}\right) \frac{E_{0}\left(P_{j}\right)}{\pi} d P_{j},
\end{aligned}
$$

we can write:

$$
L\left(P_{i}\right)=\sum_{n=1}^{\infty} C_{n}\left(P_{i}\right) r^{n}
$$

By considering only the first two bounces of light, the previous equation becomes:

$$
L\left(P_{i}\right)=C_{1}\left(P_{i}\right) r+C_{2}\left(P_{i}\right) r^{2}
$$

Note that the terms $C_{n}$ depend upon direct irradiance and scene geometry. When the radiance $L\left(P_{i}\right)$ is measured twice $\left(L^{(1)}\left(P_{i}\right), L^{(2)}\left(P_{i}\right)\right)$ for the same lighting and scene geometry but for two different known albedos of the surface $\left(r_{1}, r_{2}\right)$, the following two equations (with two unknowns $C_{1}$ and $C_{2}$ ) can be written as:

$$
\begin{aligned}
& L^{(1)}\left(P_{i}\right)=C_{1}\left(P_{i}\right) r_{1}+C_{2}\left(P_{i}\right) r_{1}^{2}, \\
& L^{(2)}\left(P_{i}\right)=C_{1}\left(P_{i}\right) r_{2}+C_{2}\left(P_{i}\right) r_{2}^{2} .
\end{aligned}
$$

There are two major difficulties with these equations. The first one is how to change surface albedo easily. The second is how to determine the surface albedo.

For the first issue, the authors proposed to simulate the change of surface albedo by varying the light color, based on the idea that albedo is light spectrum dependent. However, $C_{1}$ and $C_{2}$ depend upon direct irradiance, so the previous equation solving holds only when lighting is the same. In order to keep only two unknowns in the equation, the authors calibrated the light source to calculate the ratio between the irradiance received from different light sources. For this purpose, they use a gray convex object having a wavelength-independent albedo for any light spectrum.

Assuming that the irradiance with the second lighting is $\alpha$ times the irradiance with the first one, i.e., $E_{0}^{(2)}\left(P_{1}\right)=\alpha E_{0}^{(1)}\left(P_{1}\right)$, and considering that the ratio between the two albedos, $\beta$, is known, the equations in (48) become:

$$
\begin{gathered}
L^{(1)}\left(P_{i}\right)=C_{1}\left(P_{i}\right) r_{1}+C_{2}\left(P_{i}\right) r_{1}^{2}, \\
L^{(2)}\left(P_{i}\right) / \alpha=\beta C_{1}\left(P_{i}\right) r_{1}+\beta C_{2}\left(P_{i}\right) r_{1}^{2} .
\end{gathered}
$$

Knowing $\alpha$ and $\beta$, the terms $C_{1}\left(P_{i}\right) r_{1}$ and $C_{2}\left(P_{i}\right) r_{1}^{2}$ can be calculated.

Another calibration step is needed to obtain $\beta$ by using a non-concave object of the same material, thereby having the same reflectance, as the object of the scene. This calibration step is used to calculate the ratio between image intensities corresponding to the used lights.

In this approach, the authors use two images to separate the first and second bounced light with no need to construct a light field. This method is suitable for real-time applications and can be applied for all scenes whatever the geometry is, and under any static light sources. This approach can be extended in order to separate more bounces with the condition of adding a lighting with a different SPD for each additional bounce needed. However, this algorithm is applicable only to surfaces having a uniform reflectance. In addition, two calibration steps are needed using a gray-scale sphere and a non-concave surface of the same material as the surfaces in the scene. 
Later, $\mathrm{Fu}$ et al. [13] extended this approach using fluorescing surfaces. They proposed to remove the effects of interreflections by a bi-spectral observation of fluorescent and reflective components. Their approach is based on a single image, under the assumption of available fluorescent materials.

Other Radiosity-based approaches are worth to be listed, Shimshoni et al. [21] used an inter-reflection model similar to [16] coupled with a reflectance model for specular objects to recover the shape of polyhedral objects. Stewart and Langer [22] adapted a similar interreflection model to be used for shape recovery under diffuse lighting. Chandraker et al. [23] showed that interreflection solve generalized bas-relief ambiguity in uncalibrated photometric stereo.

\subsection{Other Approaches}

There exist in the literature other approaches that tried to handle interreflections. In this section, a brief description of these approaches is given. We chose to not give a detailed presentation of these methods for mainly two reasons, either because they do not treat interreflections directly, or because they do not fit in the main domain of interest of this survey.

Based on the dichromatic reflectance model proposed by Shafer [24], Bajcsy et al. [25, 26] analyzed interreflections by dividing them into four components, interface-interface, body-interface, interface-body and body-body reflections. The authors proposed a method that can handle and separate some interreflections in a metric space of intensity, hue and saturation in order to perform color image segmentation for dielectric materials. In [27], the authors located image areas affected by interreflections in the chromaticity sphere. Given that object colors are already estimated, they found the interreflection color by plane intersection.

In spectral imaging, Tominaga et al. [17, 28] proposed to use interreflections between fluorescent objects in order to characterize the spectral image component from the observed spectral radiance. Their method is based on a two-bounce bispectral interreflection model.

In the domain of object recoloring, handling interreflections can be necessary in order to give realistic rendering. A common approach for Lambertian scenes is to estimate two intrinsic images: reflectance intrinsic image related to surface reflectance, and illumination intrinsic image related to light's SPD. Then, in order to take into consideration diffuse interreflections, Caroll et al. [29] proposed a user-assisted method to separate the illumination intrinsic image into direct and multiple indirect components. In another work, Beigpour and Van de Weijer [30] proposed to estimate Multi-illuminant Dichromatic Reflection model for a pre-segmented singlecolored object in order to deal with multiple light sources, and indirect light sources such as interreflections.

Interreflections, as well as other indirect illumination, have been also studied in the domain of 3D-scanning using structured light. Nayar et al. [31] presented a method to separate the radiance into direct and indirect components by using high frequency illumination patterns. Based on this work, many researchers [32-34] have proposed to perform scanning under indirect illumination using structured light. However, these methods may fail in case of strong interreflections, in addition they suffer from noise and need a high number of images compared to the traditional techniques. In order to handle interreflections, Couture et al. [35] used bandpass unstructured patterns, but a large number of images is still required in this approach. Recently, Gupta et al. [36] analyzed the error in structured lights introduced by indirect illumination and proposed to combine different binary structured light pattern, each resilient to an individual indirect illumination in order to obtain a 3D scanning system applicable in the presence of a broader range of indirect illumination while needing fewer images compared to the previous approaches.

\section{Spectral Infinite-Bounce Model}

In this section, we propose an original spectral infinitebounce model which is more general than the ones presented in Section 3 and Table 1. It relates directly a physical model of interreflection to image values. This model is defined based on radiometric concepts. First, the continuous model is presented. Then, we introduce the model after surface sampling yielding to a matrix formalism. Finally, a generalization of this model, in order to take into consideration all wavelengths, is given.

\subsection{Bases of the model}

As seen before, the total irradiance in the scene is the sum of direct irradiance and indirect irradiance (irradiance after the first bounce of light, the second one, the third one and so on..). The total irradiance in a given position $P_{1}$, received after one bounce of light from all other points $P_{i}$ of the surface, can be written as:

$E_{1}\left(P_{1}\right)=\int_{P_{i} \in \mathcal{S}} r_{i} \frac{E_{0}}{\pi} K\left(P_{i}, P_{1}\right) d P_{i}$,

where the definition of geometrical kernel, $K\left(P_{i}, P_{1}\right)$, is given by equation (26). 
Similarly, taking into consideration the second bounce of light from all points $P_{j}$, we can write:

$E_{2}\left(P_{1}\right)=\int_{P_{i} \in \mathcal{S}} \int_{P_{j} \in \mathcal{S}} r_{i} r_{j} \frac{E_{0}}{\pi^{2}} K\left(P_{j}, P_{i}\right) K\left(P_{i}, P_{1}\right) d P_{j} d P_{i}$,

and for the third bounce:

$$
\begin{array}{r}
E_{3}\left(P_{1}\right)=\int_{\mathcal{S}} \int_{\mathcal{S}} \int_{\mathcal{S}} r_{i} r_{j} r_{k} \frac{E_{0}}{\pi^{3}} K\left(P_{k}, P_{j}\right) K\left(P_{j}, P_{i}\right) \\
K\left(P_{i}, P_{1}\right) d P_{k} d P_{j} d P_{i},
\end{array}
$$

and so on.

The drawback of the continuous model is that the equations contain integrals which cannot be analytically computed in the general case. By sampling the surface into a finite number of facets as proposed by Nayar et al. [6], a discrete version of this model can be obtained, providing a simpler mathematical formalism.

By sampling the surface into $m$ infinitesimal facets, the irradiance after first, second and third bounces can be written as:

$$
\begin{aligned}
& E_{1}\left(P_{1}\right)=\sum_{i \in \mathcal{S}} r_{i} \frac{E_{0}}{\pi} K\left(P_{i}, P_{1}\right) d P_{i}, \\
& E_{2}\left(P_{1}\right)=\sum_{i \in \mathcal{S}} \sum_{j \in \mathcal{S}} r_{i} r_{j} \frac{E_{0}}{\pi^{2}}, K\left(P_{j}, P_{i}\right) K\left(P_{i}, P_{1}\right) d P_{i} d P_{i},
\end{aligned}
$$

$$
\begin{array}{r}
E_{3}\left(P_{1}\right)=\sum_{i \in \mathcal{S}} \sum_{j \in \mathcal{S}} \sum_{k \in \mathcal{S}} r_{i} r_{j} r_{k} \frac{E_{0}}{\pi^{3}} K\left(P_{k}, P_{j}\right) K\left(P_{j}, P_{i}\right) \\
K\left(P_{i}, P_{1}\right) d P_{k} d P_{j} d P_{i} .
\end{array}
$$

Both irradiance and reflectance are considered to be constant over each facet.

Note that $K\left(P_{i}, P_{j}\right)$ is the element $(i, j)$ of matrix $\mathbf{K}$ presented earlier in equation (33). Note also that $r_{i}$ is the element $(i, i)$ of the matrix $\mathbf{R}$ presented previously in equation (34). Then, the irradiance vector, corresponding to the surface irradiance in its $m$ facets, after $n$ bounces of light can be written as:

$\mathbf{E}=\sum_{b=0}^{b=n}(\mathbf{K R})^{b} \mathbf{E}_{\mathbf{0}}$
Note that the division on $\pi$ has been included inside K.

This sum is a geometric series, which, when $n$ goes to infinity, converges to:

$\mathbf{E}=(\mathbf{I}-\mathbf{K R})^{-1} \mathbf{E}_{\mathbf{0}}$.

The convergence is guaranteed and can be proved physically. The energy after each bounce decreases for Lambertian nonfluorescent surfaces. This is a general equation of irradiance after infinite bounces of light for a Lambertian scene. This equation is a function of wavelength.

The total irradiance $\mathbf{E}$ is unknown and cannot be measured easily, in opposition to the total radiance. Hence, equation (57) can be written in terms of total radiance as:

$$
\begin{aligned}
\mathbf{L} & =\frac{1}{\pi} \mathbf{R}(\mathbf{I}-\mathbf{K R})^{-1} \mathbf{E}_{\mathbf{0}} \\
& =\frac{1}{\pi}\left(\mathbf{R}^{-1}-\mathbf{K}\right)^{-1} \mathbf{E}_{\mathbf{0}} .
\end{aligned}
$$

\subsection{Relation with Nayar's model [6]}

At first sight, equation (58) seems different from (36) proposed by Nayar et al. [6] (cf. section 3.3.2). However, by developing equation (36) in order to write it in term of irradiance, we can show that they are equivalent:

$\frac{1}{\pi} \mathbf{R E}=(\mathbf{I}-\mathbf{R K})^{-1} \frac{1}{\pi} \mathbf{R} \mathbf{E}_{\mathbf{0}}$.

Then,

$$
\begin{aligned}
\mathbf{E} & =\mathbf{R}^{-1}(\mathbf{I}-\mathbf{R K})^{-1} \mathbf{R} \mathbf{E}_{\mathbf{0}} \\
& =((\mathbf{I}-\mathbf{R K}) \mathbf{R})^{-1} \mathbf{R} \mathbf{E}_{\mathbf{0}} \\
& =\left(\left(\mathbf{R}^{-1}(\mathbf{I}-\mathbf{R K}) \mathbf{R}\right)\right)^{-1} \mathbf{E}_{\mathbf{0}} \\
& =(\mathbf{I}-\mathbf{K R})^{-1} \mathbf{E}_{\mathbf{0}}
\end{aligned}
$$

\subsection{Generalization to Spectral Model}

In a vision system, the radiance is captured by camera sensors, and is converted to image intensity values based on sensors' response functions as follows:

$\rho_{=}^{j} \int_{\lambda_{1}}^{\lambda_{l}} C^{j}(\lambda) L(\lambda) d \lambda$.

By sampling the spectrum, this equation can be written in a matrix form: 


$$
\boldsymbol{\rho}=\mathbf{C L}_{\lambda}
$$

where $\rho$ is a column vector containing the sensor values of a pixel, $\mathbf{C}$ is the camera response matrix of size $s \times l$ where $s$ is the number of channels and $l$ is the number of wavelengths, and $\mathbf{L}_{\lambda}$ is a column vector containing the radiance values at all different wavelengths.

Let us note that the previous equation is defined for one pixel and all wavelengths. However, the equation of radiance with interreflection introduced earlier is defined for one wavelength and for all pixels in order to take into consideration the geometrical kernel. Thus, in order to be able to combine the two equations, we need to extend the matrices to take into consideration both all pixels and all wavelengths.

We propose to extend the vector $\rho$ to the vector $\rho_{\text {ext }}$ whose size is $m s \times 1$, where $m$ is the number of pixels in the image, and whose form is:

$$
\boldsymbol{\rho}_{\text {ext }}=\left[\begin{array}{llllllllll}
\rho_{1}^{1} & . . & \rho_{m}^{1} & \rho_{1}^{2} & . . & \rho_{m}^{2} & \ldots & \rho_{1}^{b} & . . & \rho_{m}^{b}
\end{array}\right]^{T} .
$$

The vector of irradiance $\mathbf{E}_{\mathbf{0}}$ is extended in a similar way. The extended form, $\mathbf{E}_{\mathbf{0} e x t}$, has the size of $m l \times 1$ by concatenating the $\mathbf{E}_{\mathbf{0}}$ vector for each wavelength one after the other.

The reflectance matrix $\mathbf{R}$ is extended to the square diagonal matrix $\mathbf{R}_{e x t}$ whose size is $m l \times m l$. As $\mathbf{R}$ is defined for one wavelength, we can name it $\mathbf{R}_{\lambda}$, then the extended form is a concatenation of all diagonal matrices, $\mathbf{R}_{\lambda}$, on the diagonal of the new matrix:

$\mathbf{R}_{e x t}=\left[\begin{array}{cccc}\mathbf{R}_{1} & 0 & \ldots \ldots & 0 \\ \cdot & \mathbf{R}_{2} & \ldots . & \cdot \\ . & \ldots & . & 0 \\ 0 & . & \ldots \ldots & \mathbf{R}_{l}\end{array}\right]$.

The geometrical kernel matrix $\mathbf{K}$ is extended to a $m l \times m l$ block matrix whose blocks on the diagonal are $\mathbf{K}$ and all others are zero block matrices:

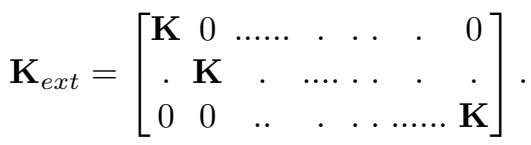

Finally, the camera response matrix is extended to the size $m s \times m l$ to take into consideration all the pixels. In order to explain the form of matrix $\mathbf{C}_{\text {ext }}$, let us introduce the matrix $\mathbf{C}^{\mathbf{i}}{ }_{\lambda}$ whose size is $m \times m$ :
$\mathbf{C}_{\lambda}^{i}=\left[\begin{array}{ccccccc}c_{\lambda_{1}}^{i} & 0 & \ldots \ldots & . & \ldots & . & 0 \\ 0 & c_{\lambda_{1}}^{i} & . & \ldots & \ldots & . & . \\ 0 & 0 & . . & . & \ldots & \ldots & c_{\lambda_{1}}^{i}\end{array}\right]$

Then, the extended matrix form of $\mathbf{C}$ can be written as:

$\mathbf{C}_{e x t}=\left[\begin{array}{ccccc}\mathbf{C}_{\lambda_{1}}^{1} & \mathbf{C}_{\lambda_{2}}^{1} & \ldots \ldots & \ldots & \mathbf{C}_{\lambda_{l}}^{1} \\ \mathbf{C}_{\lambda_{1}}^{2} & \mathbf{C}_{\lambda_{2}}^{2} & \ldots \ldots & \ldots & \mathbf{C}_{\lambda_{l}}^{2} \\ \cdot & \cdot & . & \ldots & \cdot \\ \mathbf{C}_{\lambda_{1}}^{s} & \mathbf{C}_{\lambda_{2}}^{s} & \ldots & \ldots & \mathbf{C}_{\lambda_{l}}^{s}\end{array}\right]$

After the introduction of these new matrices, a generalized spectral form of the equation (58) can be written as:

$\boldsymbol{\rho}_{e x t}=\frac{1}{\pi} \mathbf{C}_{e x t}\left(\mathbf{R}_{e x t}^{-1}-\mathbf{K}_{e x t}\right)^{-1} \mathbf{E}_{\mathbf{0 e x t}}$.

\section{Estimation of the geometrical kernel}

In this section, a more detailed study of the geometrical kernel is given. The problem of its calculation and a proposed solution are introduced.

\subsection{The problem}

The calculation of the discrete geometrical kernel presented in section 3.3.2 gives good approximation only in case that the facets $i$ and $j$ are infinitesimal and distant from each other. However, in vision systems, we are limited to the resolution of sensors when choosing the size of facets.

As the distance between the facets becomes smaller, especially when the facets are adjacent (see Figure 3), the error of calculation becomes important. Thus, calculation based on this discrete geometrical kernel model becomes false. This problem was identified by Nayar et al. [6], but no solution has been proposed.

\subsection{Monte Carlo estimation}

The definition of geometrical kernel is driven from the definition of geometrical extent, which is defined between two surfaces $\mathcal{S}_{1}$ and $\mathcal{S}_{2}$ and can be written in terms of solid angle as:

$d^{2} G=d S_{1} \cos \theta d \omega$, 


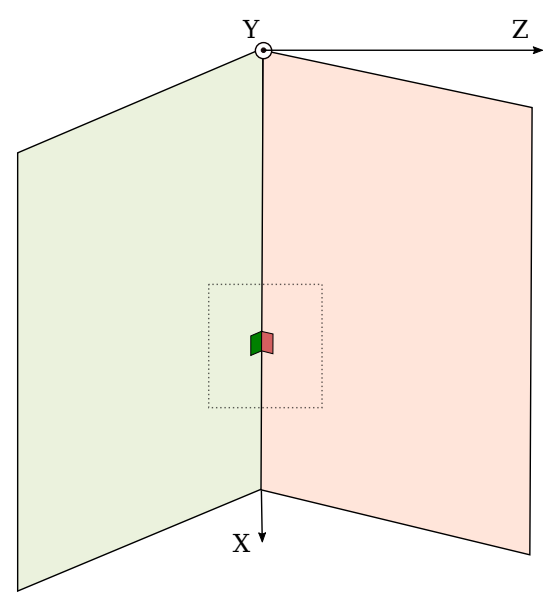

Fig. 3: Geometrical Kernel Problem: in the case of these two facets (in dark green and dark red), the error of calculation is important

where $d S_{1} \cos \theta$ is the apparent area of the infinitesimal surface $d S_{1}$, and $d \omega$ is an infinitesimal solid angle.

The function we need to estimate is then:

$G=\int_{P_{1} \in \mathcal{S}_{1}} \int_{\Omega} \cos \theta d \omega d P_{1}$,

where $\Omega$ is the set of direction where $\mathcal{S}_{2}$ is visible to $\mathcal{S}_{1}$, this can be found by projecting $\mathcal{S}_{2}$ on the unit hemisphere centered on $P_{1}$.

In order to resolve this problem we propose to estimate this integration with Monte Carlo estimator. This is done by sampling the surface of each facet and the hemisphere around the sample point: one million samples are chosen randomly over the facet, and from each sample point a ray with a random direction inside the hemisphere is traced to see if it hits the other facet. Finally, the geometrical kernel is obtained by dividing the geometrical extent on the surface of the facet.

Note that, the previous definition of geometrical extent based on solid angle is chosen for our estimator instead of the definition based on the distance between the two facets. This choice is motivated by the fact that using the distance for adjacent facets is prone to error.

\section{The Model in Practice: Experiments and Results}

We performed the experiments with two objectives in mind. The first is to study the accuracy of our model in interreflection simulation in comparison to real camera outputs. The second objective is to study the effect of using infinite bounces in comparison to using only two bounces. As seen in the state of the art, using only two bounces is very common to simplify the interreflection model $[9,10,12]$.

We divide the experiments in several sets to cover different cases of interreflection. The extensive study is performed for cases of self-interreflection and interreflection between two different surfaces for two planar Lambertian surfaces $\mathcal{S}_{1}$ and $\mathcal{S}_{2}$ having an edge contact as illustrated in Figure 4, with a known angle $\theta$ between them. Each surface has its own coordinates system, $X_{1} Y_{1}$ and $X_{2} Y_{2}$ for $\mathcal{S}_{1}$ and $\mathcal{S}_{2}$ respectively. By defining a global coordinates system $X Y Z$ as shown in Figure 4, and by performing the coordinates system changes, the geometrical kernel can be easily obtained by Monte Carlo estimation after discretization each surface into a finite set of facets. Later, other experiments handling more complicated surfaces and geometries are also shown.

In order to be able to perform these experiments, we assume that the geometrical kernel, the direct irradiance, the surface spectral reflectance, and the camera response functions are known. Then, the model can be tested for its ability to accurately simulate the camera sensor values in case of interreflection. Using Canon EOS 1000D camera with known spectral responses, we captured images, in RAW format, of the used patches under the same configuration as in simulation in order to compare simulated images to real ones. All spectral functions are taken between $400 \mathrm{~nm}$ and $700 \mathrm{~nm}$ with a a discretization step of $10 \mathrm{~nm}$. The user chose the area corresponding to a surface in the image, then this area is discretized in the same way as in the simulation. The mean value over each discrete facet is calculated and compared with the corresponding simulated value. An example of a the camera output we uses in our experiments is shown in Figure 5.

\subsection{Self-interreflection}

We chose to start with the case of self-interreflection, where interreflection happens between surfaces having the same spectral reflectance. In this case, the effect of interreflection can be easily observed and compared.

In this experience, we used six uniform colored papers, one of them is a Red Munsell paper (5R 6/12), and the other five are textile samples (see Figure 6). For each sample, we have a set of two patches of size $4 \times 4 \mathrm{~cm}$. The spectral reflectances of these papers were measured using the Minolta X-Rite Color i7. Using a platform to fix two papers on planar surfaces and to set various angles between them, we were able to capture 


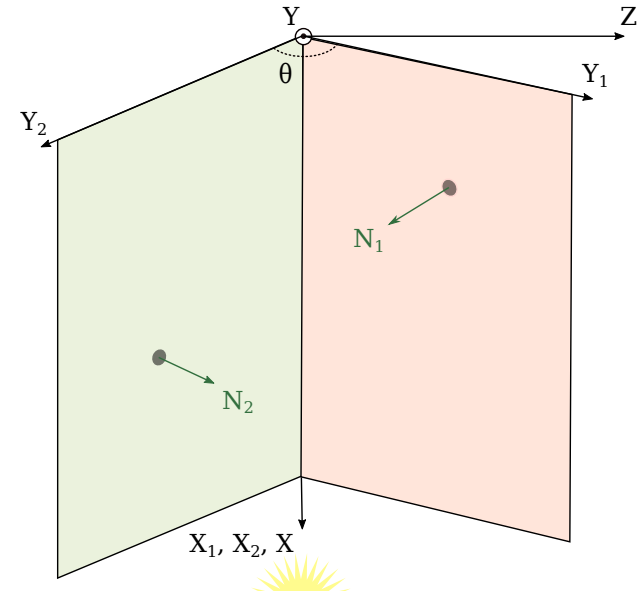

Fig. 4: First case study: two adjacent planar surfaces with a known angle $\theta$

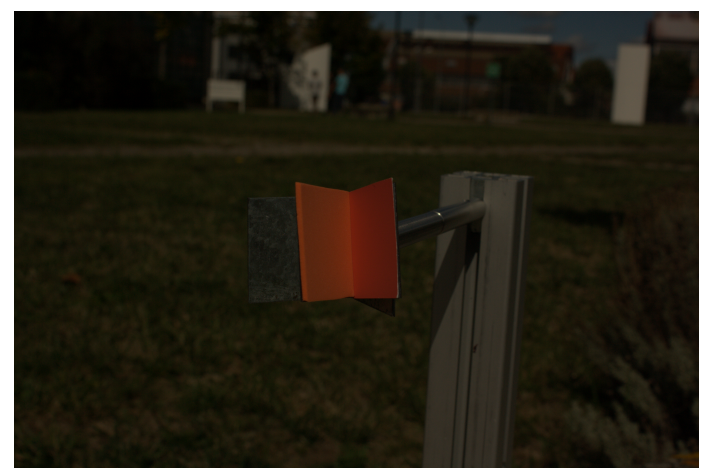

Fig. 5: An example of a camera output we used in our experiments: an orange and a Munsell red patches with an angle of $45^{\circ}$

images of interreflections between two surfaces with angles of $45^{\circ}$ and of $60^{\circ}$ between them.

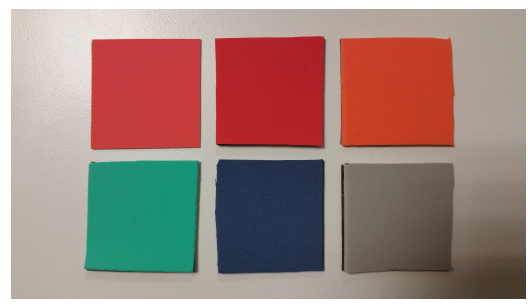

Fig. 6: The set of used samples: the first sample is a Munsell paper (5R 6/12 Mat). The other samples are textile ones.

Two different configurations are used to perform the experiments. The first is in a dark room, we use a light source to illuminate directly the platform of interreflection. The spectral power distribution of this light is measured using the Minolta cs-1000 spectroradiometer (see Figure 7). The irradiance received from direct light at each point need to be known. For this purpose, we assume having a homogeneous light source assuring that every point receives approximately the same amount of light. The second is under direct sunlight in the early afternoon, we use CIE Standard Illuminant D50 to simulate this light.

Even though we did not control all the settings in our acquisition, we were able to evaluate the ability of our model to simulate interreflections by comparing it to RGB values from real camera output.

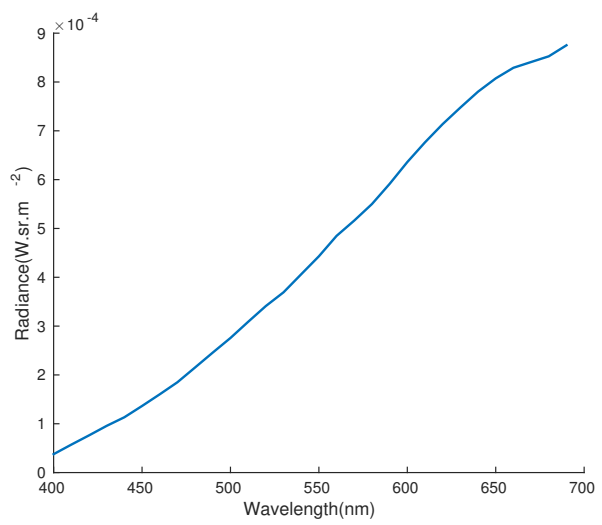

Fig. 7: Spectral Power Distribution of the lighting used in the indoor experiments.

Results We used our model to simulate infinite-bounces images for two patches of the same color with a known angle between them $\left(60^{\circ}\right.$ and $\left.45^{\circ}\right)$. In addition, to be able to see the effect of using an infinite number of bounces instead of only two, we used our model to simulate two bounce images of the two patches under the same configuration. This process was then repeated for all the patches in our sample set. The number of facets used in simulation is $8 \times 8$, where each facet is of size $5 \times 5 \mathrm{~mm}$ corresponding to approximately 2000 pixels.

Root mean square error (RMSE) values between the vector of all RGB values of camera outputs and that of the simulated image using our model with two bounces and infinite number of bounces are given in Tables 2 and 3 for angles of $45^{\circ}$ and $60^{\circ}$ respectively for the case of indoor direct light, and in Tables 4 and 5 for angles of $45^{\circ}$ and $60^{\circ}$ respectively for the case of direct sunlight.

In Figure 8, we show an example of obtained RGB values in the case of two pieces of Munsell sheets, of the same color, with an angle of $45^{\circ}$ between them. 
The values obtained from the camera output, and from simulation with infinite bounces and with two bounces are shown.
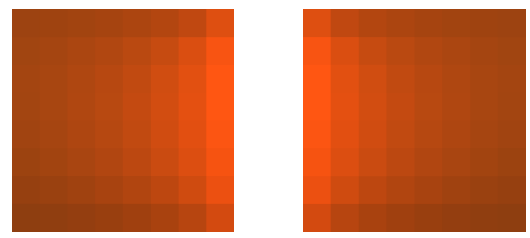

(a) With real image values

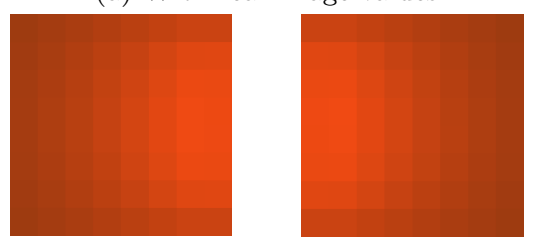

(b) With $\infty$-bounce

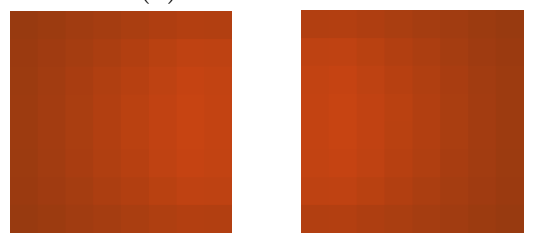

(c) With 2-bounce

Fig. 8: Real and constructed image values of two Munsell papers with an angle of $45^{\circ}$ in indoor environment under direct light.

Table 2: RMSE values between the simulated image values using 2 and $\infty$ bounces and the camera output, for self-interreflection under indoor configuration with an angle of $45^{\circ}$. Significant differences are highlighted in green when the error is reduced and in red otherwise

\begin{tabular}{|l|lll|lll|}
\hline \multirow{2}{*}{ RMSE (\%) } & \multicolumn{3}{|c|}{2 bounces } & \multicolumn{3}{c|}{$\infty$ bounces } \\
& $\mathrm{R}$ & $\mathrm{G}$ & $\mathrm{B}$ & $\mathrm{R}$ & $\mathrm{G}$ & $\mathrm{B}$ \\
\hline Red Munsell & 8.6 & 3.4 & 0.3 & 5.7 & 2.7 & 0.4 \\
\hline Red & 7.9 & 4.1 & 0.3 & 8.7 & 3.1 & 0.3 \\
\hline Orange & 9.6 & 5.9 & 0.2 & 7.2 & 4.0 & 0.3 \\
\hline Blue & 2.5 & 3.6 & 2.4 & 2.4 & 3.5 & 2.5 \\
\hline Cyan & 1.2 & 7.2 & 3.7 & 1.5 & 4.0 & 2.1 \\
\hline Gray & 4.2 & 7.3 & 1.7 & 4.2 & 5.6 & 1.5 \\
\hline
\end{tabular}

6.2 Interreflection between two surfaces with different colors

In this section, we study the case of interreflection between two surfaces with different spectral reflectance
Table 3: RMSE values between the simulated image values using 2 and $\infty$ bounces and the camera output, for self-interreflection under indoor configuration with an angle of $60^{\circ}$. Significant differences are highlighted in green when the error is reduced and in red otherwise.

\begin{tabular}{|l|lll|lll|}
\hline \multirow{2}{*}{ RMSE (\%) } & \multicolumn{3}{|c|}{2 bounces } & \multicolumn{3}{c|}{$\infty$ bounces } \\
& $\mathrm{R}$ & $\mathrm{G}$ & $\mathrm{B}$ & $\mathrm{R}$ & $\mathrm{G}$ & $\mathrm{B}$ \\
\hline Red Munsell & 7.3 & 3.5 & 0.6 & 4.6 & 2.8 & 0.6 \\
\hline Red & 6.7 & 3.5 & 0.6 & 5.4 & 3.0 & 0.7 \\
\hline Orange & 7.2 & 5.3 & 0.3 & 8.0 & 4.3 & 0.3 \\
\hline Blue & 3.2 & 4.0 & 3.2 & 3.1 & 3.9 & 3.4 \\
\hline Cyan & 1.7 & 6.2 & 6.2 & 1.6 & 5.4 & 2.1 \\
\hline Gray & 3.9 & 6.4 & 1.5 & 3.7 & 5.3 & 1.4 \\
\hline
\end{tabular}

Table 4: RMSE values between the simulated image values using 2 and $\infty$ bounces and the camera output, for self-interreflection under outdoor configuration with an angle of $45^{\circ}$. Significant differences are highlighted in green when the error is reduced and in red otherwise

\begin{tabular}{|l|lll|lll|}
\hline \multirow{2}{*}{ RMSE (\%) } & \multicolumn{3}{|c|}{2 bounces } & \multicolumn{3}{c|}{$\infty$ bounces } \\
& $\mathrm{R}$ & $\mathrm{G}$ & $\mathrm{B}$ & $\mathrm{R}$ & $\mathrm{G}$ & $\mathrm{B}$ \\
\hline Red Munsell & 10.6 & 6.5 & 1.3 & 4.7 & 4.5 & 0.9 \\
\hline Red & 6.6 & 1.2 & 2.3 & 3.1 & 1.8 & 1.4 \\
\hline Orange & 6.1 & 2.3 & 1.6 & 5.3 & 3.6 & 0.6 \\
\hline Blue & 10.0 & 2.9 & 9.9 & 9.9 & 3.1 & 9.7 \\
\hline Cyan & 3.9 & 5.4 & 5.1 & 3.1 & 1.9 & 4.4 \\
\hline Gray & 3.6 & 2.6 & 3.0 & 3.8 & 3.2 & 3.5 \\
\hline
\end{tabular}

Table 5: RMSE values between the simulated image values using 2 and $\infty$ bounces and the camera output, for self-interreflection under outdoor configuration with an angle of $60^{\circ}$. Significant differences are highlighted in green when the error is reduced and in red otherwise

\begin{tabular}{|l|lll|lll|}
\hline \multirow{2}{*}{ RMSE (\%) } & \multicolumn{3}{|c|}{2 bounces } & \multicolumn{3}{c|}{$\infty$ bounces } \\
& $\mathrm{R}$ & $\mathrm{G}$ & $\mathrm{B}$ & $\mathrm{R}$ & $\mathrm{G}$ & $\mathrm{B}$ \\
\hline Red Munsell & 11.2 & 6.7 & 3.2 & 7.7 & 5.4 & 2.1 \\
\hline Red & 8.2 & 5.9 & 1.6 & 5.4 & 4.5 & 1.0 \\
\hline Orange & 7.4 & 4.1 & 1.8 & 3.4 & 2.3 & 0.8 \\
\hline Blue & 27.4 & 7.0 & 34.1 & 27.4 & 6.9 & 33.9 \\
\hline Cyan & 2.4 & 2.5 & 3.2 & 2.0 & 3.5 & 3.3 \\
\hline Gray & 6.1 & 4.4 & 7.3 & 6.1 & 3.9 & 7.4 \\
\hline
\end{tabular}

functions. The acquisitions were done under direct sunlight whose spectral reflectance is considered that of Standard Illuminant D50 in the simulation. The angle between the two surfaces is set to $45^{\circ}$. We did two different experiments, the first is for interreflection between two colored surfaces. For this case, the patches, the sizes, and all the other settings are exactly the same as explained in the previous section. The second is for interreflection between one colored surface and another white one. Both surfaces are of the size of 
$9 \mathrm{~cm} \times 6 \mathrm{~cm}$, each is discretized into 100 facets of the size of $9 \mathrm{~mm} \times 6 \mathrm{~mm}$. For the colored surfaces we used the same set of patches as before. The white patch is the White Balance Chart of the ColorChecker Passport.

Results in terms of RMSE values between camera output and simulation are shown in Table 6 for interreflection between two-colored surfaces and between one white and one colored surfaces.

Table 6: RMSE values between the simulated image values using 2 and $\infty$ bounces and the camera output, for interreflection between two different surfaces under outdoor configuration with an angle of $45^{\circ}$. Significant differences are highlighted in green when the error is reduced and in red otherwise

\begin{tabular}{|l|lll|lll|}
\hline \multirow{2}{*}{ RMSE (\%) } & \multicolumn{3}{|c|}{ 2 bounces } & \multicolumn{3}{c|}{$\infty$ bounces } \\
& $\mathrm{R}$ & $\mathrm{G}$ & $\mathrm{B}$ & $\mathrm{R}$ & $\mathrm{G}$ & $\mathrm{B}$ \\
\hline Red Munsell & 15.9 & 2.2 & 1.7 & 4.1 & 1.2 & 1.9 \\
Orange & 5.7 & 7.6 & 3.1 & 9.5 & 5.6 & 2.8 \\
\hline Red Munsell & 8.2 & 3.0 & 4.3 & 5.8 & 2.5 & 5.0 \\
Cyan & 1.1 & 2.5 & 3.6 & 1.1 & 3.7 & 3.1 \\
\hline Red Munsell & 4.7 & 3.1 & 2.3 & 5.9 & 3.1 & 2.8 \\
Blue & 5.3 & 5.4 & 8.9 & 5.9 & 5.7 & 8.6 \\
\hline White & 0.8 & 7.3 & 7.9 & 2.6 & 5.5 & 4.4 \\
Cyan & 2.2 & 10.1 & 10.4 & 2.3 & 5.9 & 6.0 \\
\hline White & 1.7 & 4.8 & 4.4 & 5.8 & 3.6 & 3.5 \\
Red & 18.8 & 4.8 & 4.6 & 10.5 & 5.4 & 4.9 \\
\hline
\end{tabular}

6.3 Interreflection with different geometries and surfaces

In this section, we present some examples of interreflection simulation with different geometries and more than two surfaces with different spectral reflectance. The first example is for two planar surfaces each of two colors with an angle of $45^{\circ}$ between them (see Figure (9)). The acquisition is done under direct sunlight. Each surface is of the size of $8 \mathrm{~cm} \times 4 \mathrm{~cm}$ and is discretized into 100 facets of size $8 \mathrm{~mm} \times 4 \mathrm{~mm}$. The shared edge between the surfaces is along the longest dimension.

RMSE values are shown in Table 7 for simulation with infinite bounces and with two bounces for each uniformly colored surfaces.

The second example is with a geometry of the shape $\mathrm{W}$ where the central surfaces are shallower than the exterior ones (see Figure (10)). The exterior surfaces are both of a size of $10 \mathrm{~cm} \times 8 \mathrm{~cm}$ and are discretized each to 200 facets of the size of $10 \mathrm{~mm} \times 4 \mathrm{~mm}$. The interior surfaces are of a size of $10 \mathrm{~mm} \times 4 \mathrm{~mm}$ and are discretized each to 100 facets of the size of $10 \mathrm{~mm} \times 4 \mathrm{~mm}$. The shared edge between the surfaces is along the longest

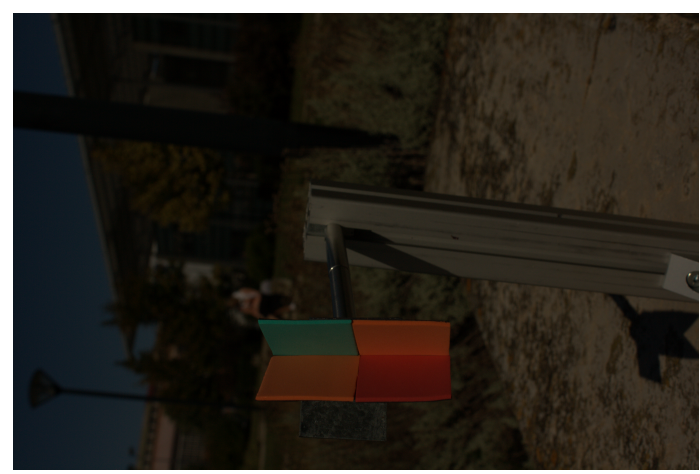

Fig. 9: The camera output we used in our experiments for surfaces with multiple spectral reflectances: orangered and cyan-orange surfaces with an angle of $45^{\circ}$

Table 7: RMSE values between the simulated image values using 2 and $\infty$ bounces and the camera output, for interreflection between two different surfaces each of two colors under outdoor configuration with an angle of $45^{\circ}$. Significant differences are highlighted in green when the error is reduced and in red otherwise

\begin{tabular}{|l|lll|lll|}
\hline \multirow{2}{*}{ RMSE (\%) } & \multicolumn{3}{|c|}{2 bounces } & \multicolumn{3}{c|}{$\infty$ bounces } \\
& $\mathrm{R}$ & $\mathrm{G}$ & $\mathrm{B}$ & $\mathrm{R}$ & $\mathrm{G}$ & $\mathrm{B}$ \\
\hline Orange 1 & 4.4 & 12.0 & 1.1 & 8.7 & 9.7 & 1.4 \\
Red & 19.9 & 1.8 & 4.7 & 8.0 & 2.4 & 4.9 \\
Cyan & 2.3 & 4.6 & 2.5 & 1.5 & 3.4 & 1.9 \\
Orange 2 & 8.3 & 8.9 & 0.6 & 7.4 & 6.7 & 0.6 \\
\hline
\end{tabular}

dimension. The acquisitions here also are done under direct sunlight.

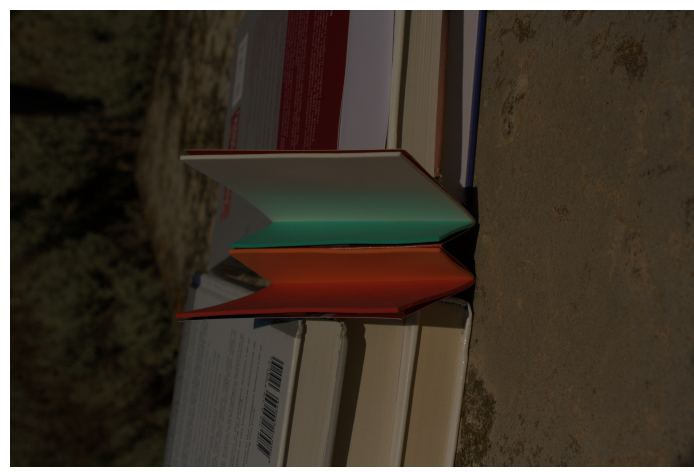

Fig. 10: The camera output we used in our experiments for surfaces with geometry showing occlusions: W shape with red, orange, cyan and white surfaces. The angles are approximately $45^{\circ}$

RMSE values are shown in Table 8 for simulation with infinite bounces and with two bounces for each uniformly colored surfaces. 
Table 8: RMSE values between the simulated image values using 2 and $\infty$ bounces and the camera output, for interreflection between surfaces of a W shape under outdoor configuration. Significant differences are highlighted in green when the error is reduced and in red otherwise

\begin{tabular}{|l|lll|lll|}
\hline \multirow{2}{*}{ RMSE (\%) } & \multicolumn{3}{|c|}{2 bounces } & \multicolumn{3}{c|}{$\infty$ bounces } \\
& $\mathrm{R}$ & $\mathrm{G}$ & $\mathrm{B}$ & $\mathrm{R}$ & $\mathrm{G}$ & $\mathrm{B}$ \\
\hline Red & 18.5 & 7.1 & 5.2 & 10.7 & 5.1 & 5.3 \\
Orange & 11.7 & 2.9 & 5.3 & 6.0 & 4.4 & 2.7 \\
Cyan & 1.8 & 9.5 & 5.6 & 1.8 & 7.1 & 4.4 \\
White & 3.9 & 14.2 & 8.5 & 2.4 & 8.6 & 5.8 \\
\hline
\end{tabular}

\subsection{Discussion}

From the previous tables, we can observe that there is sometimes no significant enhancement when using an infinite-bounce model in comparison to a two-bounce model. However, the smaller the angle the stronger the enhancement. This is especially true in case of outdoor configuration where the homogeneity of illuminant can be guaranteed. In the case of Lambertian surfaces, which is almost the case for the Munsell sheets, RMSE values are always smaller when using our infinite-bounce model. At first sight, it seems that the assumption that it is enough to take into consideration only two bounces of light, thus only one bounce of interreflection, is true. However, if we look at the RSME values taken for the closet facets to the joint edge between the patches, the difference between RMSE values when simulating with infinite bounces and with two bounces becomes important. In Table 9, the evolution of RMSE values with number of bounces in indoor configuration is shown. In this table, RMSE values are calculated using only the first column of facets; it is the closest to the shared edge, thus the most subject to interreflection. The effect of interreflections is very important in this area and decreases when getting farther from the joint edge. The RMSE values are shown for the channel where the error is the highest. For Munsell sheets, the error is tripled when using only two bounces. The error decreases when adding extra bounces.

Notice that this observation is true only in case of a surface with a high reflectance (the surface spectral reflectance function shows high values). For surfaces with low reflectance, the error difference is negligible. For example, for the blue patch, the error in all cases is small (see Table 9). The blue patch as a much lower reflectance that the Munsell patch, as shown in Figure 11.

Figure (12) shows the evolution of RMSE values with the column number starting from the shared edge

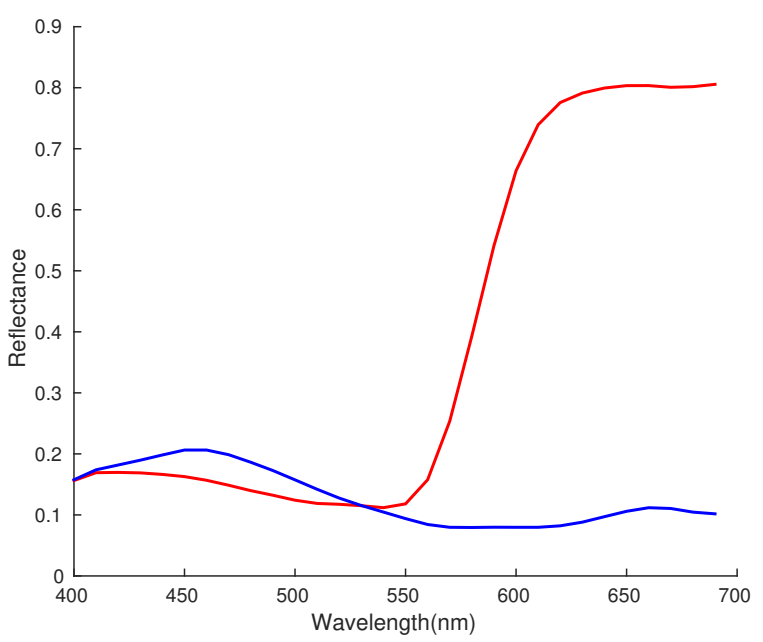

Fig. 11: Spectral reflectance functions: Munsell patch in red, blue patch in blue.

for the case of self-interreflection with an angle of $45^{\circ}$ of Munsell Sheet.

We also compared the radiances after two bounces and infinity of bounces. Figure (13a) shows that radiance after infinite bounces of interreflection carries more energy compared to that after one bounce of interreflection for a facet close to the shared edge. When a facet which is far from this edge, the difference between the two radiances becomes small, (13b).

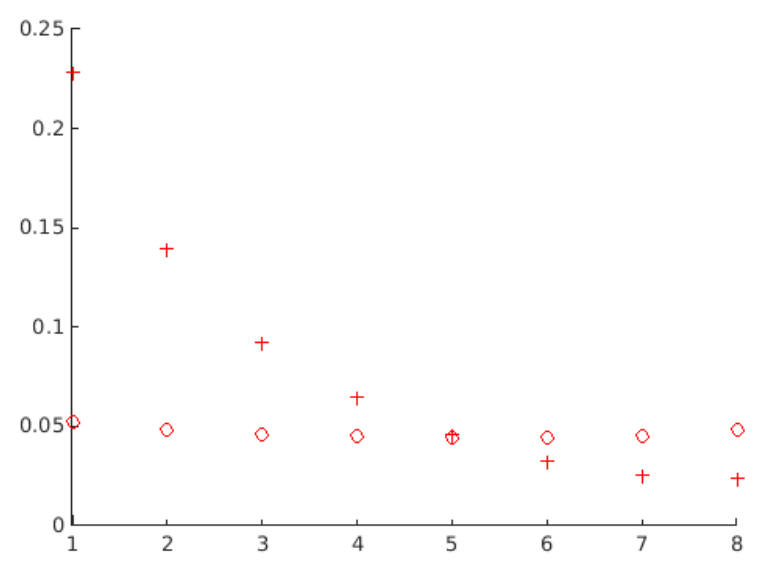

Fig. 12: RMSE values with column number starting from the shared edge between: circle for infinite bounce, cross for 2 bounces.

Sources of error The model we proposed is driven from a physical background which guarantees its accuracy in 
Table 9: RMSE between the simulated image values of the column closest to the joint edge and those of the camera output with an angle of $45^{\circ}$

\begin{tabular}{|l|l|l|l|l|l|}
\hline RMSE (\%) & Color Channel & 2 Bounces & 3 Bounces & 4 Bounces & $\infty$ Bounces \\
\hline Munsell & $\mathrm{R}$ & 20.4 & 12.0 & 8.8 & 6.7 \\
\hline Red & $\mathrm{R}$ & 22.2 & 14.9 & 12.1 & 10.3 \\
\hline Orange & $\mathrm{R}$ & 21.4 & 11.7 & 7.6 & 4.5 \\
\hline Blue & $\mathrm{B}$ & 2.0 & 1.7 & 1.7 & 1.7 \\
\hline
\end{tabular}

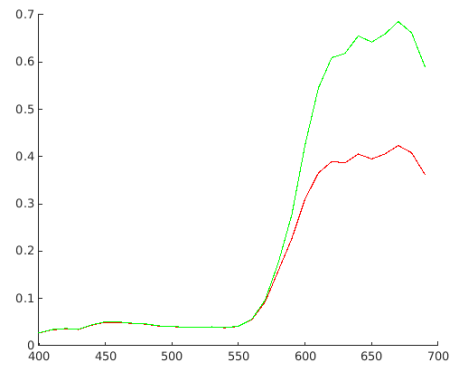

(a) Center facet in the column closest to the joint edge

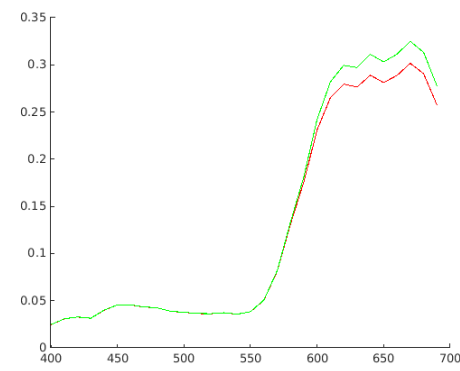

(b) Center facet in the column farthest to the joint edge

Fig. 13: Radiance after two bounces, in red, and infinite number of bounces, in green of interreflection for Munsell red patch with an angle of $45^{\circ}$.

the continuous domain and we have seen in Tables 2 to 6 that the error in simulation compared to the image is less than $6 \%$ in most of the tests. Although this error value can be considered as neglectable in most of the computer vision applications, it is important to have an idea about the reasons explaining this error. In the following, a discussion about some possible sources of this error is presented.

- Non homogeneity of light: The illuminant used in our indoor experiments is an optical fiber behind a diffusing disk, which illuminates directly the platform. Even though we have considered the light to be perfectly diffuse and homogeneous, in reality it is not the case. Therefore, using a flat white surface, we performed a study on the homogeneity of this illuminant.

We measured the spectral power distribution of light on different places on a region of the white surface corresponding to the size of our samples. The coefficient of variation of the RMSE between different measurements is $1.4 \%$. However, as the direct irradiance received on a surface element depends on its distance from the light source, variations in irradiance per surface element are important especially in our experiments where surfaces are close to the light source. We calculated the coefficient of variation of RMSE values for two different distances between the white surface and the light source. The value we obtained is $14 \%$. The two distances correspond to the closest and farthest edges of the used interreflection platform. In addition, we calculated the RMSE values between image intensities taken for the white surface and its mean value. The RMSE values are $0.025,0.031$ and 0.013 for R, G and B channels respectively. However, these values do not correspond solely to the non-homogeneity of lighting, they are related also to image noise introduced by the camera sensors. Thus, these values may vary when the settings of camera change.

The non-homogeneity of the indoor illuminant explain that the results in outdoor configuration are better. However, ambient light is not taken into consideration in outdoor simulation, which plays a role too in increasing the error.

- Surface defects: the textile surfaces we have used are not totally homogeneous. They show a texture in them which introduces an error in the measurements. Moreover, neither the size nor the angle are totally controlled, which contributes to the error we obtained.

- Facets size: the physical model is accurate in continuous space. When passing to discrete space, the error is increased with the size of the facets. However, in our case, doubling the number of facets reduced the error of simulation of only $0.2 \%$ this probably due to the noisy nature of the used camera outputs. The choice of the number of facets is an important trade-off between the processing time, the accuracy, 
and the sensors noise. For most vision application, reasonably big facets can be the best choice.

Limitations One of the possible limitations of the proposed model is the rapid increase of the number of facets needed to to simulate interreflection with good accuracy with the increase of complexity of geometry or the spectral reflectances of the surface. This leads to extremely big matrices and to a high complexity, memory usage and processing time. One solution that we are investigating is to use sparse matrices due to the fact that the matrices used contains a large number of zeros. Even though the accuracy is theoretically increased with the number of facets, image values are more prone to sensor noise which introduces errors in the simulation compared to real camera outputs.

\section{Conclusion}

In this paper, we have presented existing approaches in the state of the art in the domain of interreflection modeling for computer vision applications. Different classifications of these methods have been introduced regarding the number of bounces and the number of color channels or wavelengths used, as well as regarding the preconditions needed for each approach to perform properly. Preconditions can be light related, surface related or any dependency on other method or on calibration steps.

Moreover, we introduced a new model, a spectral infinite-bounce interreflection model, inspired by the radiosity equation. In this approach, a physical model of interreflection, taking into consideration an infinite number of bounces, is directly related to RGB values of an image. Complex Lambertian surfaces, with a different spectral reflectance per surface element, can be handled. Our results proved the importance of the use of infinite number of bounces to model interreflection especially for areas close to edges where adjacent elements can see each others. This model, being more general than those of the state of the art, can open, we believe, a new possibility in front of the research in this area.

We aim to use this model in order to solve the inverse problem in computer vision. It can be used in spectral reflectance estimation, in illuminant SPD estimation or in color calibration. In addition, we believe that this model can be used in combination with pattern recognition approaches in domains such as deep color constancy, image colorization, style transfer, etc. However, an extension to non-Lambertian surfaces can be important in order to be able to handle real-life images.

\section{References}

1. M. F. Cohen and D. P. Greenberg, "The hemi-cube: A radiosity solution for complex environments," in ACM SIGGRAPH Computer Graphics, vol. 19, pp. 31-40, ACM, 1985.

2. H. W. Jensen, Realistic image synthesis using photon mapping, vol. 364. Ak Peters Natick, 2001.

3. M. Pharr, W. Jakob, and G. Humphreys, Physically based rendering: From theory to implementation. Morgan Kaufmann, 2016.

4. D. Forsyth and A. Zisserman, "Mutual illumination," in Computer Vision and Pattern Recognition, 1989. Proceedings CVPR'89., IEEE Computer Society Conference on, pp. 466473, IEEE, 1989.

5. D. Forsyth and A. Zisserman, "Shape from shading in the light of mutual illumination," Image and vision computing, vol. 8, no. 1, pp. 42-49, 1990.

6. S. K. Nayar, K. Ikeuchi, and T. Kanade, "Shape from interreflections," International Journal of Computer Vision, vol. 6, no. 3, pp. 173-195, 1991.

7. M. S. Drew and B. V. Funt, "Calculating surface reflectance using a single-bounce model of mutual reflection," in Computer Vision, 1990. Proceedings, Third International Conference on, pp. 394-399, IEEE, 1990.

8. J. Ho, B. V. Funt, and M. S. Drew, "Separating a color signal into illumination and surface reflectance components: Theory and applications," IEEE Transactions on Pattern Analysis and Machine Intelligence, vol. 12, no. 10, pp. 966-977, 1990.

9. B. V. Funt, M. S. Drew, and J. Ho, "Color constancy from mutual reflection," International Journal of Computer Vision, vol. 6, no. 1, pp. 5-24, 1991.

10. B. V. Funt and M. S. Drew, "Color space analysis of mutual illumination," Pattern Analysis and Machine Intelligence, IEEE Transactions on, vol. 15, no. 12, pp. 1319-1326, 1993.

11. S. M. Seitz, Y. Matsushita, and K. N. Kutulakos, "A theory of inverse light transport," in Computer Vision, 2005. ICCV 2005. Tenth IEEE International Conference on, vol. 2, pp. 1440-1447, IEEE, 2005.

12. M. Liao, X. Huang, and R. Yang, "Interreflection removal for photometric stereo by using spectrum-dependent albedo," in Computer Vision and Pattern Recognition (CVPR), 2011 IEEE Conference on, pp. 689-696, IEEE, 2011.

13. Y. Fu, A. Lam, Y. Matsushita, I. Sato, and Y. Sato, "Interreflection removal using fluorescence," in European Conference on Computer Vision, pp. 203-217, Springer, 2014.

14. F. E. Nicodemus, "Radiance," American Journal of Physics, vol. 31, no. 5, pp. 368-377, 1963.

15. M. Hébert, R. D. Hersch, and P. Emmel, "Fundamentals of optics and radiometry for color reproduction," Handbook of Digital Imaging, 2015.

16. S. K. Nayar and Y. Gong, "Colored interreflections and shape recovery," in Image Understanding Workshop, pp. 333-343, 1992.

17. S. Tominaga, K. Kato, K. Hirai, and T. Horiuchi, "Spectral image analysis of mutual illumination between florescent objects," JOSA A, vol. 33, no. 8, pp. 1476-1487, 2016.

18. M. S. Drew and B. V. Funt, "Variational approach to interreflection in color images," JOSA A, vol. 9, no. 8, pp. 1255$1265,1992$.

19. J. J. Koenderink and A. Van Doorn, "Geometrical modes as a general method to treat diffuse interreflections in radiometry," JOSA, vol. 73, no. 6, pp. 843-850, 1983.

20. D. Forsyth and A. Zisserman, "Reflections on shading," IEEE Transactions on Pattern Analysis and Machine Intelligence, vol. 13, no. 7, pp. 671-679, 1991. 
21. I. Shimshoni and J. Ponce, "Recovering the shape of polyhedra using line-drawing analysis and complex reflectance models," Computer Vision and Image Understanding, vol. 65, no. 2, pp. 296-310, 1997.

22. A. J. Stewart and M. S. Langer, "Toward accurate recovery of shape from shading under diffuse lighting," IEEE Transactions on Pattern Analysis and Machine Intelligence, vol. 19, no. 9, pp. 1020-1025, 1997.

23. M. K. Chandraker, F. Kahl, and D. J. Kriegman, "Reflections on the generalized bas-relief ambiguity," in Computer Vision and Pattern Recognition, 2005. CVPR 2005. IEEE Computer Society Conference on, vol. 1, pp. 788-795, IEEE, 2005.

24. S. A. Shafer, "Using color to separate reflection components," Color Research \& Application, vol. 10, no. 4, pp. 210-218, 1985.

25. R. Bajcsy, S. Lee, and A. Leonardis, "Color image segmentation with detection of highlights and inter-reflections," in SPIE/SPSS Symposium, Santa Clara, 1990.

26. R. Bajcsy, S. W. Lee, and A. Leonardis, "Detection of diffuse and specular interface reflections and inter-reflections by color image segmentation," International Journal of Computer Vision, vol. 17, no. 3, pp. 241-272, 1996.

27. S. Tominaga and T. Okayama, "A method for analyzing color images containing highlight and interreflection effects," Systems and computers in Japan, vol. 28, no. 13, pp. 8-18, 1997.

28. S. Tominaga, K. Kato, K. Hirai, and T. Horiuchi, "Bispectral interreflection estimation of fluorescent objects," in Color and Imaging Conference, vol. 2015, pp. 111-115, Society for Imaging Science and Technology, 2015.

29. R. Carroll, R. Ramamoorthi, and M. Agrawala, "Illumination decomposition for material recoloring with consistent interreflections," in ACM Transactions on Graphics (TOG), vol. 30, p. 43, ACM, 2011.

30. S. Beigpour and J. Van de Weijer, "Object recoloring based on intrinsic image estimation," in 2011 International Conference on Computer Vision, pp. 327-334, IEEE, 2011.

31. S. K. Nayar, G. Krishnan, M. D. Grossberg, and R. Raskar, "Fast separation of direct and global components of a scene using high frequency illumination," in ACM Transactions on Graphics (TOG), vol. 25, pp. 935-944, ACM, 2006.

32. T. Chen, H. P. Lensch, C. Fuchs, and H.-P. Seidel, "Polarization and phase-shifting for $3 \mathrm{~d}$ scanning of translucent objects," in Computer Vision and Pattern Recognition, $200 \%$. CVPR'07. IEEE Conference on, pp. 1-8, IEEE, 2007.

33. T. Chen, H.-P. Seidel, and H. P. Lensch, "Modulated phaseshifting for 3d scanning," in Computer Vision and Pattern Recognition, 2008. CVPR 2008. IEEE Conference on, pp. 18, IEEE, 2008.

34. J. Gu, T. Kobayashi, M. Gupta, and S. K. Nayar, "Multiplexed illumination for scene recovery in the presence of global illumination," in Computer Vision (ICCV), 2011 IEEE International Conference on, pp. 691-698, IEEE, 2011.

35. V. Couture, N. Martin, and S. Roy, "Unstructured light scanning to overcome interreflections," in Computer Vision (ICCV), 2011 IEEE International Conference on, pp. 18951902, IEEE, 2011.

36. M. Gupta, A. Agrawal, A. Veeraraghavan, and S. G. Narasimhan, "A practical approach to $3 \mathrm{~d}$ scanning in the presence of interreflections, subsurface scattering and defocus," International journal of computer vision, vol. 102, no. 1-3, pp. 33-55, 2013. 